Research Article

\title{
Optimization of Damage Equivalent Accelerated Test Spectrum Derivation Using Multiple Non-Gaussian Vibration Data
}

\author{
Fei Xu $\left(D,{ }^{1}\right.$ Kjell Ahlin, ${ }^{2}$ and Binyi Wang ${ }^{3}$ \\ ${ }^{1}$ School of Automotive Engineering, Yancheng Institute of Technology, Yancheng 224051, China \\ ${ }^{2}$ Professor Emeritus Blekinge Tekniska Högskola, 37179 Karlskrona, Sweden \\ ${ }^{3}$ Northwest Institute of Mechanical and Electrical Engineering, Xianyang 712000 Shaanxi, China \\ Correspondence should be addressed to Fei Xu; luoyefeihen@163.com
}

Received 10 July 2021; Accepted 15 August 2021; Published 6 September 2021

Academic Editor: Nan Li

Copyright (c) 2021 Fei Xu et al. This is an open access article distributed under the Creative Commons Attribution License, which permits unrestricted use, distribution, and reproduction in any medium, provided the original work is properly cited.

The response spectra are widely used in the damage assessment of non-Gaussian random vibration environments and the derivation of damage equivalent accelerated test spectrum. The effectiveness of the latter is strongly affected by modal parameter uncertainties, multiple field data processing, and the nonsmooth shape of the derived power spectral density (PSD). Optimization of accelerated test spectrum derivation based on dynamic parameter selection and iterative update of spectrum envelope is presented in this paper. The extreme response spectrum (ERS) envelope of the field data is firstly taken as the limiting spectrum, and the corresponding relationship between damping coefficient, fatigue exponent, and damage equivalent PSD under different test times is constructed to achieve the dynamic selection of uncertain parameters in the response spectrum model. Then, an iterative update model based on the weighted sum of fatigue damage spectrum (FDS) error is presented to reduce the error introduced by the nonsmooth shape of the derived PSD. The case study shows that undertest can be effectively avoided by the dynamic selection of model parameters. The weighted error is reduced from $80.1 \%$ to $7.5 \%$ after 7 iterations. Particularly, the error is close to 0 within the peak and valley frequency band.

\section{Introduction}

Random vibration tests are widely used to identify defects in product design and to evaluate the reliability and fatigue life of products. To simulate the mechanical environment experienced by products during their life cycle in the lab, the test spectrum is usually derived from the field measured data, which is collected using different sensors (e.g., ultrasonic transducer and accelerometer) [1]. With multiple and complicated field data, the accuracy of signal processing algorithms and data analysis methods are key factors when understanding the mechanical environment and facilitating the test spectrum derivation.

Power spectral density (PSD) is used by typical digital shaker controllers to describe the random vibration environment experienced by products, with an implicit assumption that the vibration data follows a Gaussian distribution. How- ever, field recorded time histories, e.g., wind-induced vibration data [2] and road roughness-induced vehicle vibration data [3], usually present non-Gaussian characteristics. Since non-Gaussian (especially super-Gaussian) random vibration tends to shorten the fatigue life of products $[4,5]$, different vibration control methods were studied to simulate the non-Gaussian vibration environment in the lab. Steinwolf presented a phase manipulation method to simulate the non-Gaussian data [6]. In particular, the selected phase is transformed from random to deterministic in order to obtain a prescribed kurtosis. PSD and probability density function (PDF) are controlled independently. An analytical relation between kurtosis, amplitude, and phase at specific frequencies was presented later to make this method applicable in a closed-loop control [7]. From the perspective of time-varying PSD and PDF, a non-stationary nonGaussian stochastic process simulation method based on 
the zero-memory nonlinear translation relationship between non-Gaussian and Gaussian stochastic processes is proposed by Cui et al. [8]. Fei et al. [9] presented a method to synthesize non-Gaussian random vibration that is characterized by running RMS (root mean square). The essential idea is to model the non-Gaussian signal by a Gaussian signal multiplied by an amplitude modulation function (AMF). A twoparameter Weibull distribution is used to model the PDF of the running RMS and to create the AMF. Zheng et al. [10] presented a control method for multi-input multioutput stationary non-Gaussian random vibration test using timedomain randomization. The frequency-domain inverse system method is utilized to obtain the desired drive signals for dynamic inputs, and the skewness, kurtosis, and PSD of response signals are controlled efficiently and simultaneously.

The major drawback of these non-Gaussian vibration control methods, when used for fatigue life evaluation of high reliability and long-life products, is the long test time and high cost. To address this problem, the response spectrum method was utilized to evaluate the potential damage introduced by the vibration environment. An accelerated test spectrum was then synthesized from the response spectra based on the damage equivalence criterion [11-13]. To perform life cycle potential damage assessment and test tailoring, Lalanne [14] derived an equivalent PSD from multiple task profiles based on the frequency domain fatigue damage spectrum (FDS). Wijker [15] and Decker et al. [16] derived the accelerated test spectrum based on the extreme response spectrum (ERS). The effect of parameter uncertainties (damping coefficient and fatigue exponent) on the root mean square (RMS) value of the derived PSD was studied. Steinwolf and Wolfsteiner [17] indicated that to avoid changes in the failure mechanism and dynamic properties of products, the ERS of field data should be used to limit the accelerated PSD level. Xu et al. [18] showed that the FDS of non-Gaussian field data increases as the kurtosis increases. A significant error would be introduced if FDS and ERS were calculated in the frequency domain directly using PSD. Ahlin [19] calculated FDS and ERS in time domain using the ramp invariant digital filter method. Due to the low computational efficiency of FDS in the time domain, Wolfsteiner [20] decomposed the non-Gaussian load into a combination of Gaussian loads, so that the frequency domain method can be used to improve the computational efficiency. Assuming that the synthesized equivalent PSD is flat within the half-power bandwidth, Cianetti et al. [21] presented the equivalent PSD synthesis method for nonGaussian data using FDS and Mile's Equation. These existing methods have demonstrated how to derive the equivalent accelerated test spectrum from a single set of nonGaussian data and analyzed the influence of parameter uncertainties on the RMS value of test spectrum. However, how to select the correct model parameters using multiple non-Gaussian vibration data, so as to derive the equivalent accelerated test spectrum which can effectively avoid undertest, has not been studied. In addition, how to construct the objective function, so as to rapidly reduce the error introduced by nonsmooth shape of the synthesized PSD, has not been demonstrated. To address these problems, an optimization design procedure of test spectrum derivation based on dynamic parameter selection and iterative spectrum envelope update technique is presented in this paper.

The remainder of the paper is organized as follows. Section 2 introduces the definition of Gaussian random vibration, non-Gaussian random vibration, FDS, and ERS. The optimized accelerated test spectrum derivation procedure is also presented. In Section 3, validation with field measured non-Gaussian vibration data is presented. Finally, the discussion and conclusions are summarized in Section 4.

\section{Materials and Methods}

2.1. Gaussian and Non-Gaussian Random Vibration. For the field measured discrete random vibration signal $x(t)$, the higher-order moments can be used to determine if it follows the Gaussian distribution:

$$
m_{n}=\int_{-\infty}^{\infty} x^{n} p(x) d x=\frac{1}{N} \sum_{j=1}^{N} x_{j}^{n},
$$

where $m_{n}$ is the $n$th order moments, $p(x)$ is the probability density function (PDF), and $N$ is the number of signals.

When the mean value is zero,

$$
\begin{aligned}
& S=\frac{1}{N} \sum_{j=1}^{N} \frac{x_{j}^{3}}{\sigma_{x}^{3}}=\frac{m_{3}}{\sigma_{x}^{3}}, \\
& K=\frac{1}{N} \sum_{j=1}^{N} \frac{x_{j}^{4}}{\sigma_{x}^{4}}=\frac{m_{4}}{\sigma_{x}^{4}},
\end{aligned}
$$

where $S$ is the skewness, $K$ is the kurtosis, and $\sigma_{x}$ is the root mean square (RMS) of $x(t)$.

For a Gaussian distributed signal, the skewness is 0 and the kurtosis is 3. Deviation from these values indicates that the signal follows a non-Gaussian distribution. From the perspective of response spectrum calculation of a nonGaussian signal, kurtosis is more important than skewness, since it represents the probability of peak values in time history [22].

\subsection{Fatigue Damage Spectrum (FDS) and Extreme Response} Spectrum (ERS). The FDS and ERS are widely used response spectra for evaluating potential damage under random vibration environment. The FDS is essentially a plot that shows the response of a series of single-degree-of-freedom (SDOF) systems to the base input acceleration time history. Many SDOF systems tuned to a range of natural frequencies are assessed using the same input. The FDS shows the fatigue damage encountered for a particular SDOF system anywhere within the analyzed time. It has been shown that the stress is roughly proportional to pseudovelocity [23]. For a SDOF system with a natural frequency $f_{n}$ and a damping ratio $\xi$, the output pseudovelocity $x_{\mathrm{pv}}$ to an input 
acceleration $x_{a}$ can be calculated using a ramp invariant digital filter method [19]:

$$
\begin{aligned}
x_{\mathrm{pv}} & =F_{\text {filter }}\left(b, a, x_{a}\right), \\
a & =\left[1,-2 C, E^{2}\right], \\
b & =\frac{-1}{w 2 \pi f_{n}}\left[\frac{C-1}{Q}+q S+w,-2 C w+\frac{1-E^{2}}{Q}-2 q S, E^{2}\left(w+\frac{1}{Q}\right)-\frac{C}{Q}+q S\right] \\
w & =\frac{2 \pi f_{n}}{f_{s}} \\
Q & =\frac{1}{2 \zeta}, \\
k_{1} & =\sqrt{1-\frac{1}{4 Q^{2}}} \\
k_{2} & =\frac{\pi}{Q f_{s}}, \\
k_{3} & =\frac{2 \pi}{f_{s}} k_{1}, \\
q & =\frac{(1 / 2 / Q / Q-1)}{k_{1}}, \\
A & =k_{2} f_{n}, \\
B & =k_{3} f_{n}, \\
E & =\exp (-A), \\
C & =E \cos (B), \\
S & =E \sin (B), \\
&
\end{aligned}
$$

where $f_{s}$ is the sampling frequency and $F_{\text {filter }}$ indicates filtering the input signal with a ramp invariant digital filter.

With the output $x_{\mathrm{pv}}$, the cumulative damage can be calculated in both time domain and frequency domain. In time domain, the rain flow cycle counting method is usually used for stress cycle counting. With cyclic numbers of different stress levels, the S-N curve and Miner's rule are combined to calculate the fatigue damage as follows [24]:

$$
\begin{gathered}
N_{i}=c S_{i}^{-b}, \\
D_{t}=\sum_{i=1}^{p} n_{i} / N_{i}=\sum_{i=1}^{p} n_{i} k^{b} x_{\mathrm{pv}, i}^{b} / c=k^{b} / c * \sum_{i=1}^{p} n_{i} x_{\mathrm{pv}, i}^{b},
\end{gathered}
$$

where $N_{i}$ is the fatigue life at stress $S_{i}(i=1,2, \cdots, p), p$ is the number of stress levels considered, $n_{i}$ is the number of cycle exposure at $S_{i}, c$ is a constant, $b$ is the fatigue exponent, $k$ is the proportional coefficient of stress to pseudovelocity, $D_{t}$ is the total damage index calculated in time domain, and $x_{\mathrm{pv}, i}$ is the output pseudovelocity at ith cycle.

In the frequency domain, Rayleigh distribution of response stress maxima is assumed and used to calculate

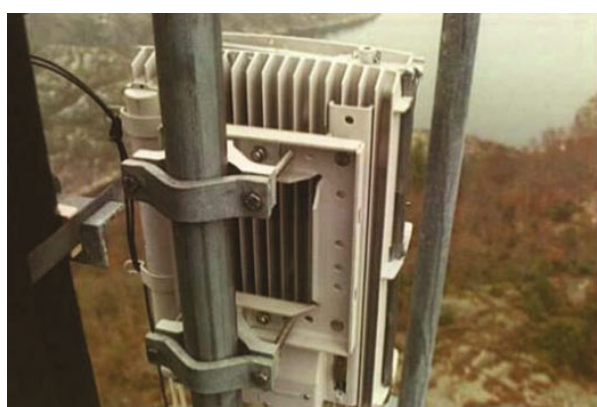

Figure 1: Test item.

the stress cycles:

$$
p(S)=\frac{S}{\sigma_{S}^{2}} e^{-S^{2} / 2 \sigma_{S}^{2}},
$$

where $S$ is the stress value of peaks and $\sigma_{S}$ is the RMS of the stress time history.

The total damage can be calculated as

$$
D_{f}=\frac{f_{n} T}{c} \int_{0}^{+\infty} p(S) S^{b} d S,
$$

where $T$ is the total time of exposure to the stress environment.

Substituting Equation (5) into Equation (6) leads to

$$
D_{f}=\frac{f_{n} T}{c} k^{b}\left(2 \sigma_{p v}^{2}\right)^{b / 2} \Gamma\left(1+\frac{b}{2}\right),
$$

where $\Gamma$ is the gamma function and $\sigma_{\mathrm{pv}}$ is the RMS of pseudovelocity.

The RMS of pseudovelocity can be calculated using

$$
\sigma_{\mathrm{pv}}=\sqrt{\sum_{f=f_{\mathrm{low}}}^{f=f_{\mathrm{hi}}}\left(|H(f)|^{2} * G_{x x}(f)\right) * d f},
$$

where $H(f)$ is the transmissibility of a SDOF system (pseudovelocity/acceleration), $G_{x x}(f)$ is the input PSD, $f_{\text {low }}$ is the lower limit, and $f_{\text {hi }}$ is the higher limit.

If the input PSD is relatively flat in the half-power bandwidth of each SDOF system, then Mile's Equation can be used to calculate $\sigma_{\mathrm{pv}}$ in a closed form approximately as follows:

$$
\sigma_{\mathrm{pv}}=\sqrt{\frac{G_{x x}\left(f_{n}\right) Q}{8 \pi f_{n}}} .
$$

With Equations (7) and (9), the PSD at each natural frequency can be derived from the FDS at the corresponding 

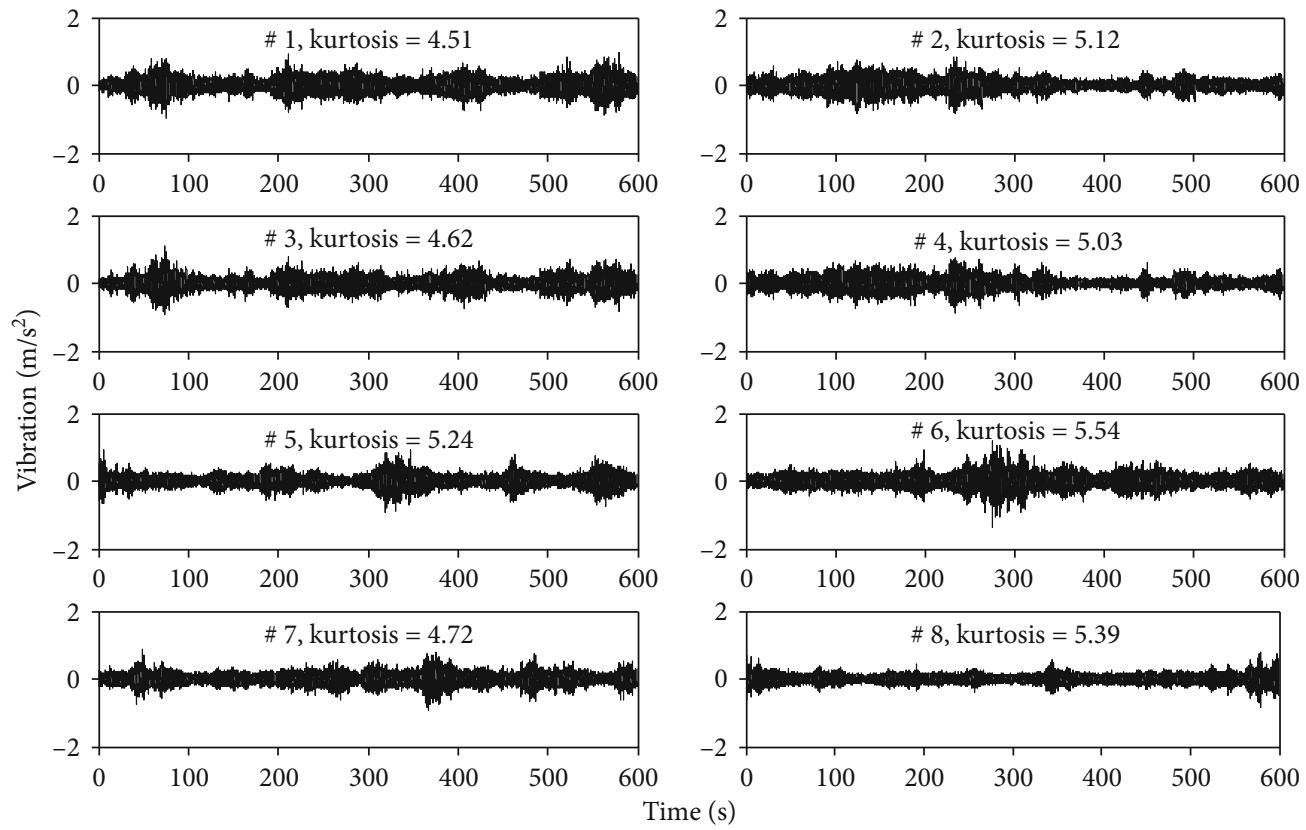

Figure 2: Field measured non-Gaussian signal induced by wind.

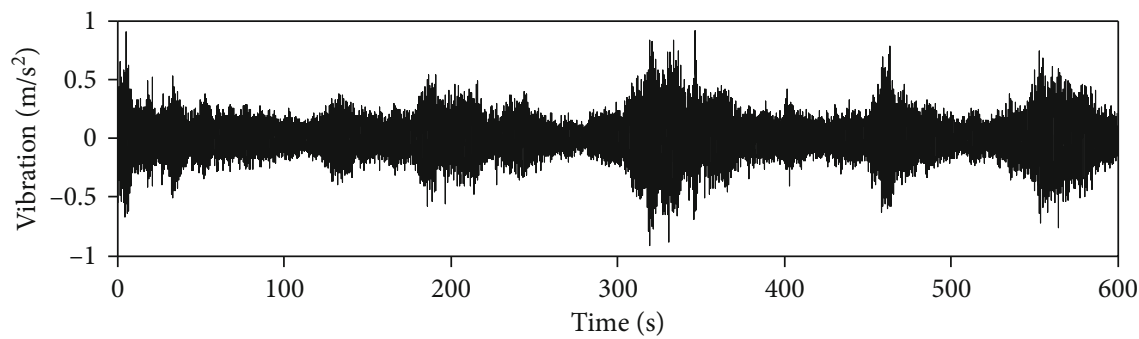

— Time history of non-Gaussian signal \#5

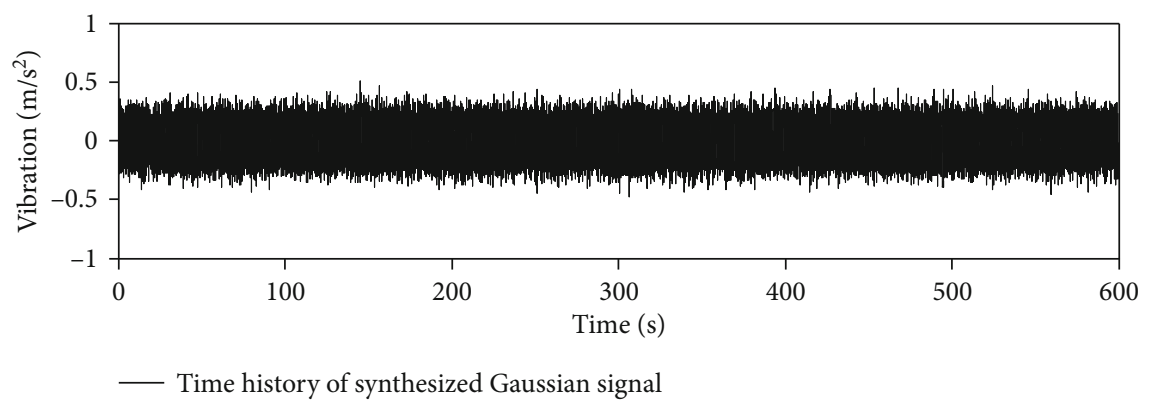

Figure 3: Comparison of time history between the measured non-Gaussian signal and the synthesized Gaussian signal.

natural frequency:

$$
G_{x x}\left(f_{n}\right)=\left(\frac{D\left(f_{n}\right)}{\Gamma(1+b / 2) f_{n} T}\right)^{2 / b} \frac{4 \pi f_{n}}{Q}
$$

Note that if the flat PSD assumption does not hold around some natural frequencies, the FDS calculated with Equations (7) and (8) using the derived PSD in Equation (10) will deviate from the FDS calculated with Equations
(7) and (9). In such case, an iterative update procedure is needed to take the shape of PSD into account (see Section 4).

The ERS shows the damage caused by vibration from an overstress viewpoint. Like the FDS, the ERS is essentially a plot that shows the responses of a number of SDOF systems to a base input acceleration time history. The only difference is that the ERS is generated by calculating the maximum response of a SDOF system to the input. The final plot, the ERS, shows the largest response encountered for a particular SDOF system anywhere within the analyzed time. For a 


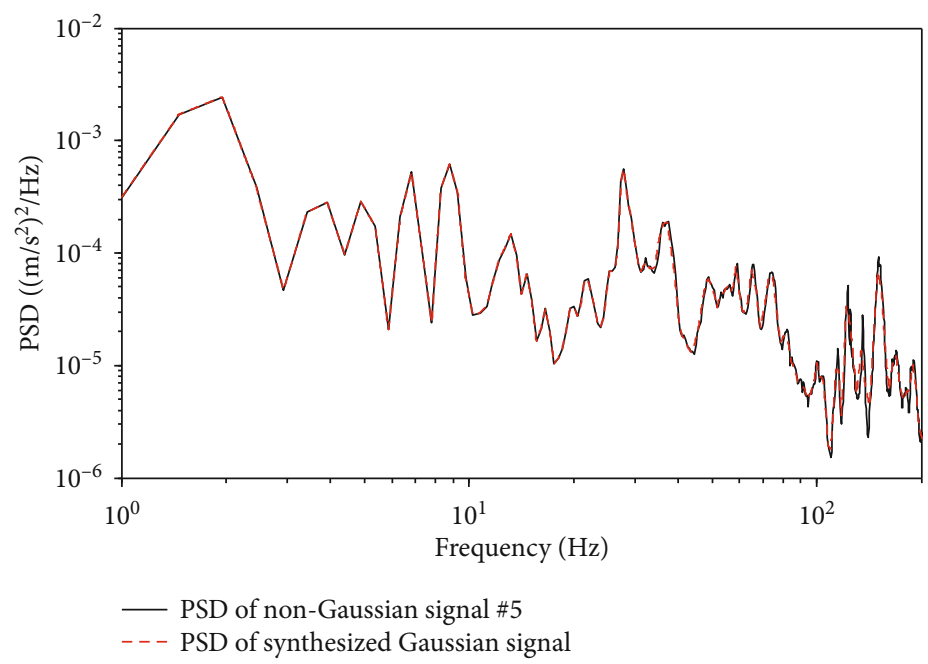

Figure 4: Comparison of PSD between the measured non-Gaussian signal and the synthesized Gaussian signal.

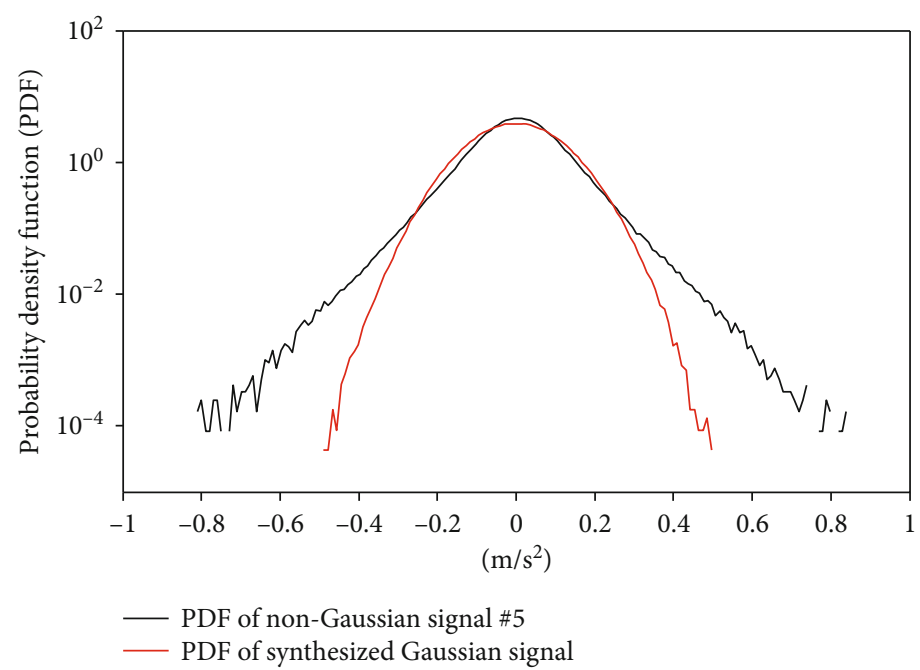

Figure 5: Comparison of PDF between the measured non-Gaussian signal and the synthesized Gaussian signal.

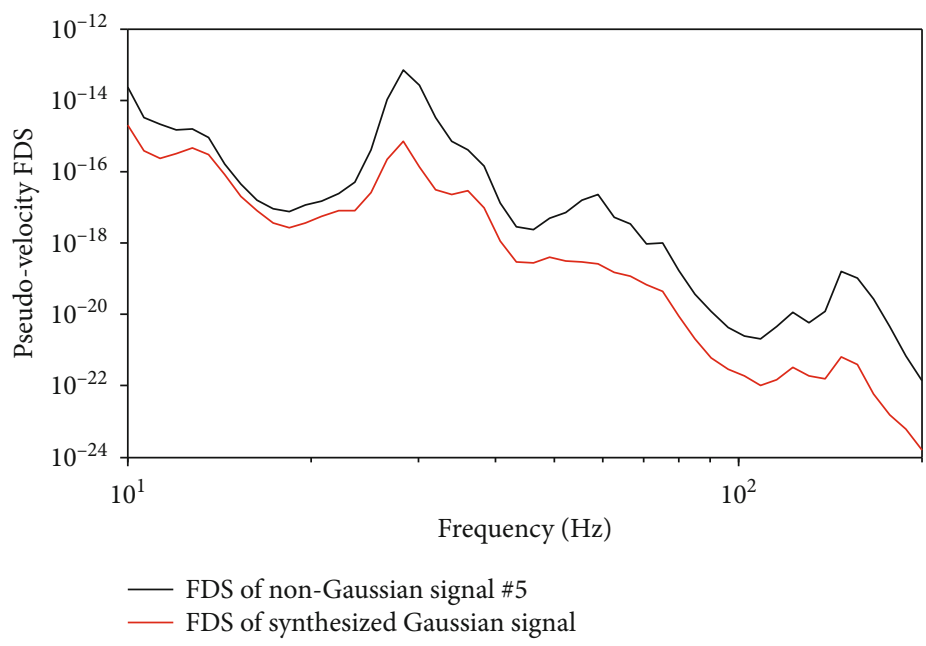

FIGURE 6: Comparison of FDS between the measured non-Gaussian signal and the synthesized Gaussian signal. 

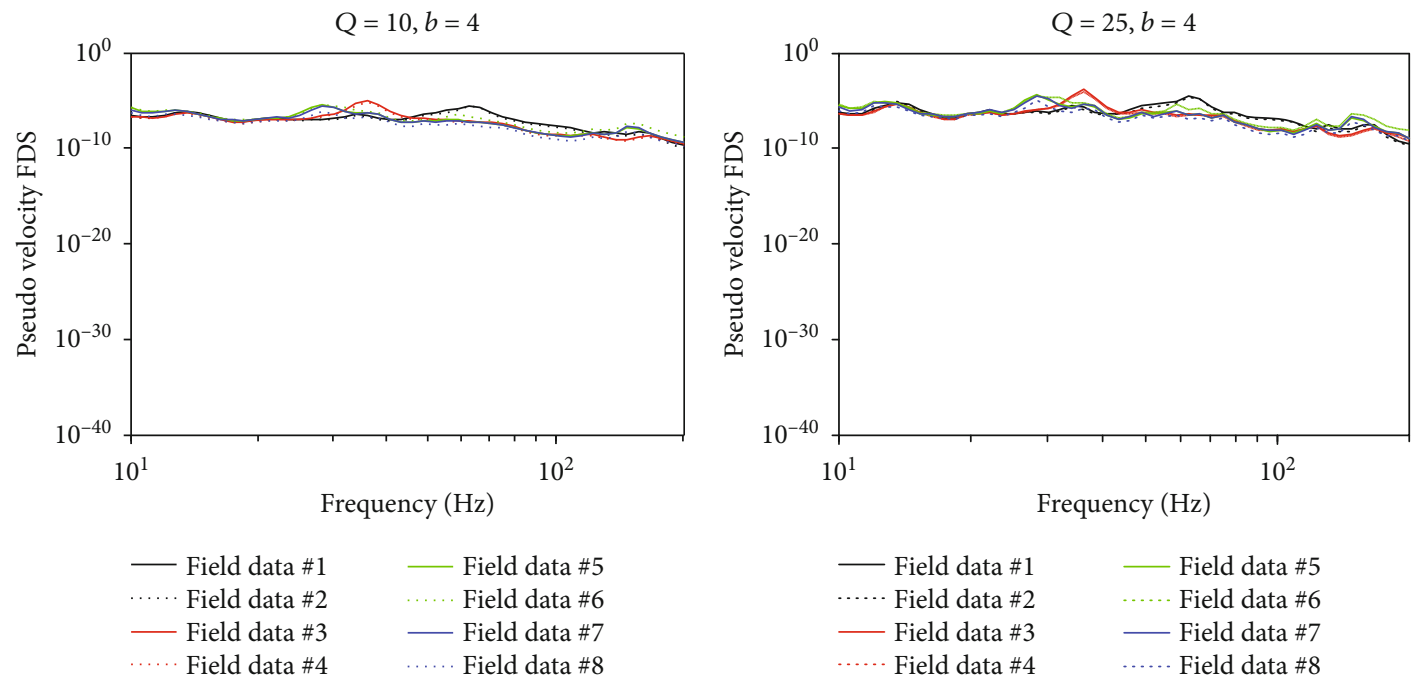

(a)

(b)
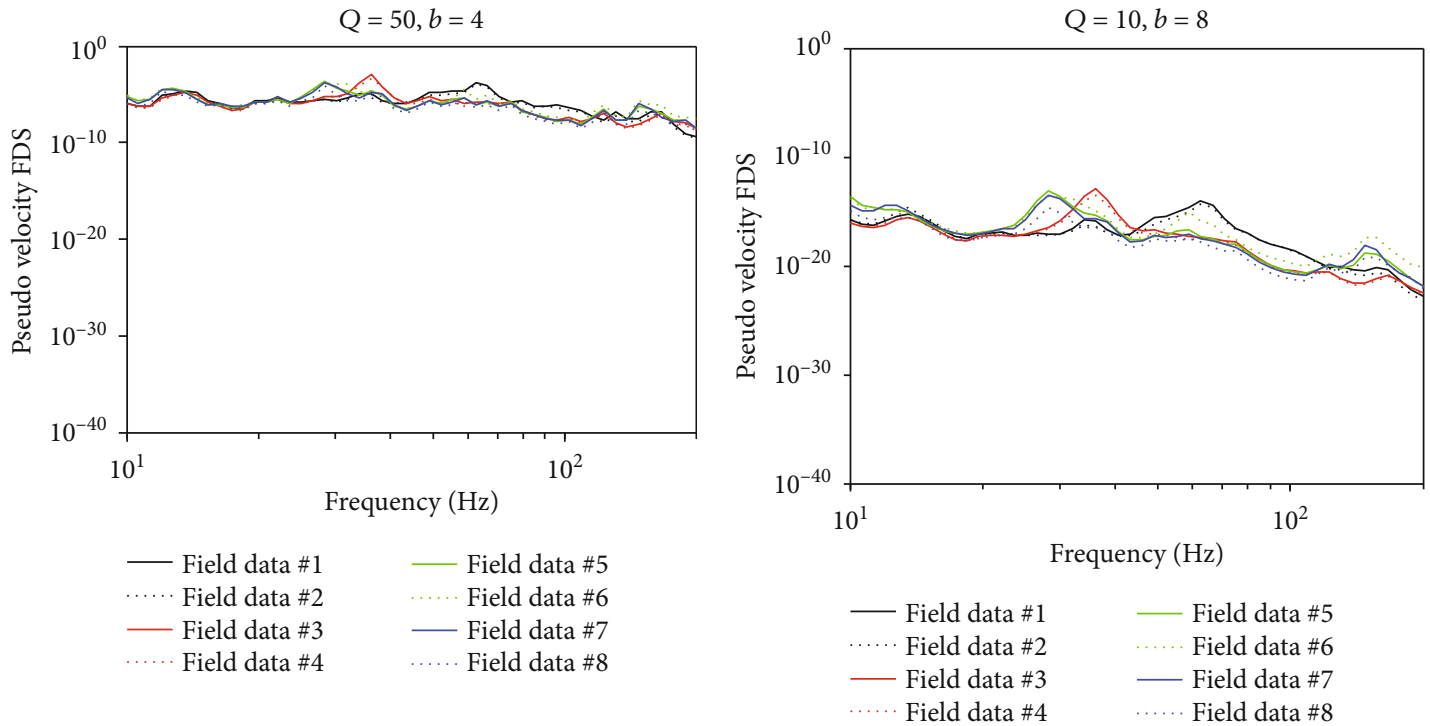

(c)

(d)

Figure 7: Continued. 


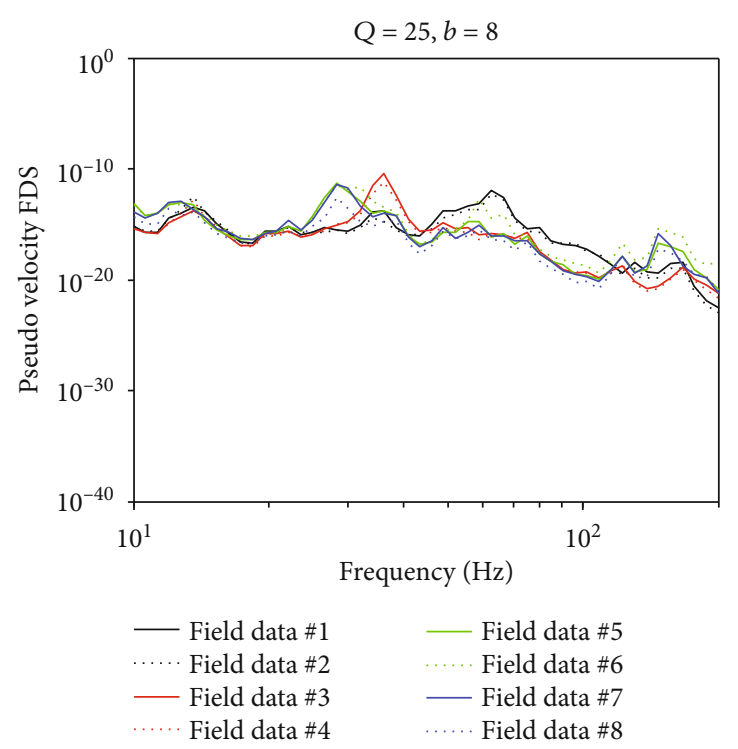

(e)

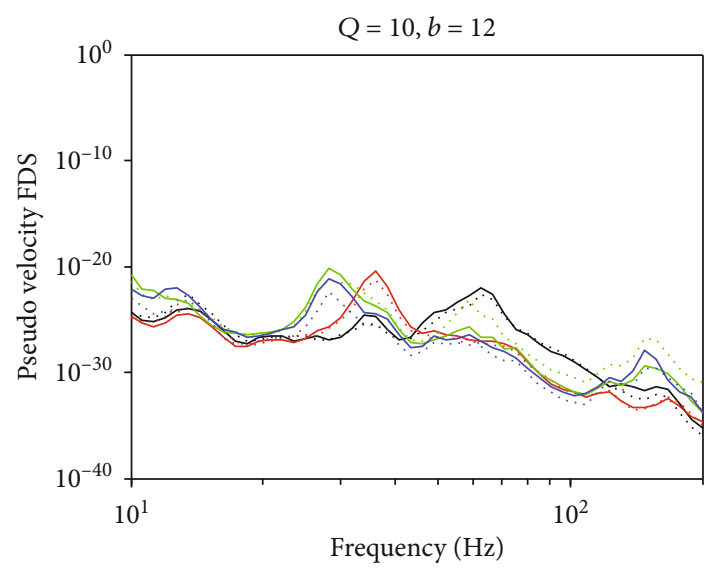

Frequency $(\mathrm{Hz})$

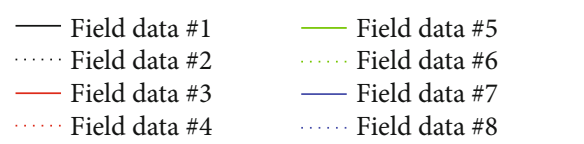

(g)

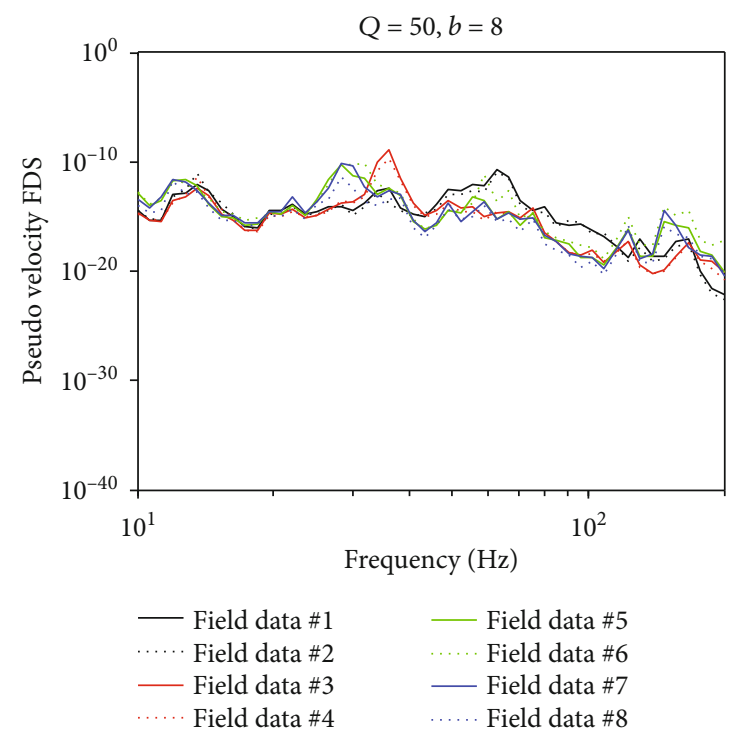

(f)

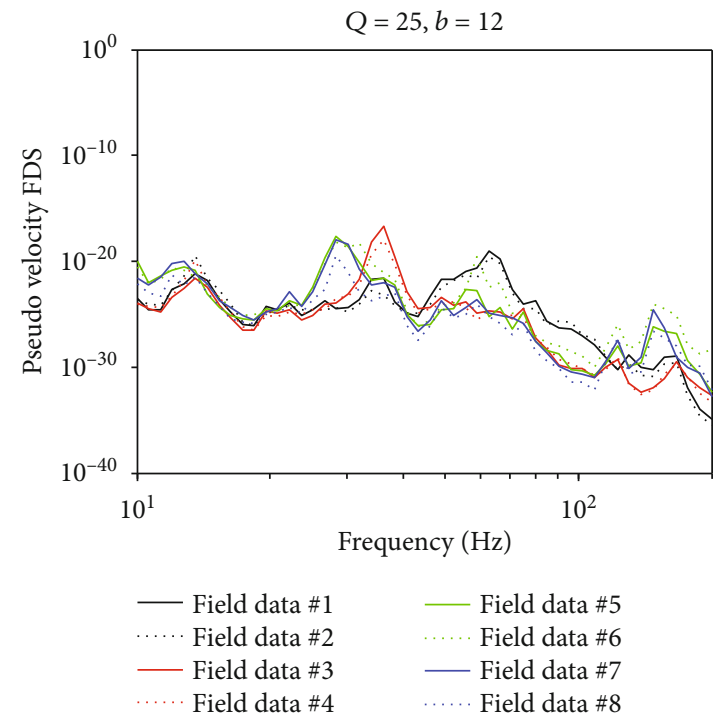

(h)

Figure 7: Continued. 


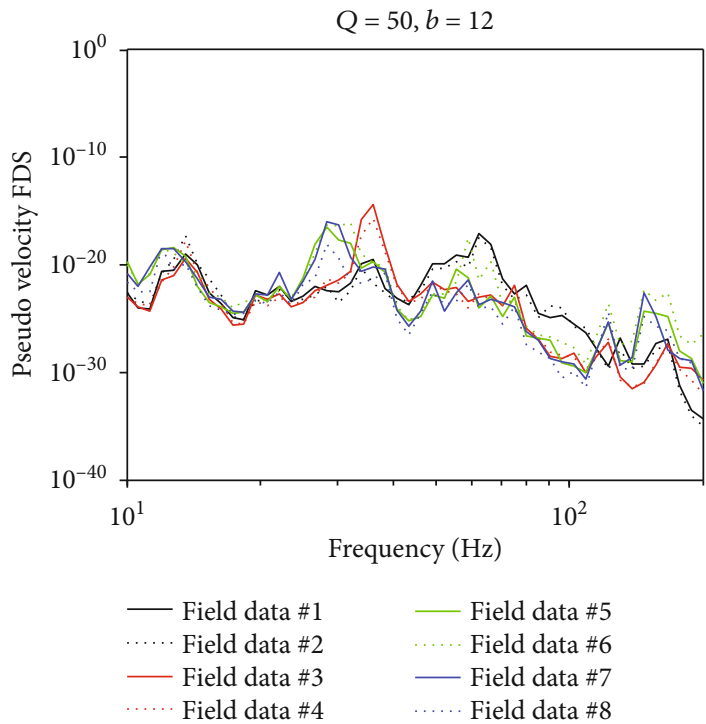

(i)

Figure 7: FDS of measured wind load under different $Q$ and $b$ values: (a) $Q=10, b=4$; (b) $Q=25, b=4$; (c) $Q=50, b=4$; (d) $Q=10, b=8$; (e) $Q=25, b=8$; (f) $Q=50, b=8$; (g) $Q=10, b=12$; (h) $Q=25, b=12$; (i) $Q=50, b=12$.

SDOF system with a natural frequency $f_{n}$ and a damping ratio $\xi$, the ERS can also be calculated in both time domain and frequency domain as follows [14]:

$$
\begin{gathered}
\operatorname{ERS}_{t}=\max \left(F_{\text {filter }}\left(b, a, x_{a}\right)\right), \\
\text { ERS }_{f}=\left(\sqrt{2 \ln f_{n} * T}+\frac{0.577}{\sqrt{2 \ln f_{n} * T}}\right) \\
* \sqrt{\sum_{f=f_{\text {low }}}^{f=f_{\text {hi }}}\left(H(f)^{\wedge} 2 * G_{x x}(f)\right) * d f} .
\end{gathered}
$$

2.3. Derivation of Damage Equivalent Accelerated Test Spectrum. Since the accuracy of the damage equivalent accelerated test spectrum is strongly affected by uncertain parameters, dispersion of multiple non-Gaussian samples, and the smoothness of the derived PSD, an optimum design procedure is presented as follows:

(1) Calculate the FDS of multiple samples in time domain and take the envelope using different damping ratios (denoted by quality factor $Q$ ) and fatigue exponent $b$

(2) Predetermine the accelerated test time, and synthesize the initial PSD using the FDS envelope and Equation (10)

(3) Choose $Q$ and $b$ in a dynamic way, so that the most conservative PSD is derived

(4) Calculate the FDS in the frequency domain using the derived PSD, Equation (7), and Equation (8)
(5) Compare the calculated FDS in Step (1) and Step (4), and define the weighted sum of FDS errors:

$$
\begin{aligned}
W_{\text {error }}= & \sum_{f_{i}=f_{\text {lowest }}}^{f_{\text {hiest }}}\left[\left|\frac{D_{\text {num }}\left(f_{n}\right)-D_{\text {env }}\left(f_{n}\right)}{D_{\text {env }}\left(f_{n}\right)}\right|\right. \\
& \left.* 100 * \frac{D_{\text {env }}\left(f_{n}\right)}{\sum_{f_{i}=f_{\text {lowest }}}^{f_{\text {liest }}} D_{\text {env }}\left(f_{n}\right)}\right],
\end{aligned}
$$

where $f_{\text {lowest }}$ is the lowest natural frequency, $f_{\text {hiest }}$ is the highest natural frequency, $D_{\text {env }}\left(f_{n}\right)$ is the FDS envelope calculated in Step (1), and $D_{\text {num }}\left(f_{n}\right)$ is the FDS calculated in Step (4)

(6) Update the PSD in Step (3) until a certain error is reached:

$$
G_{m+1}\left(f_{n}\right)=G_{m}\left(f_{n}\right) *\left(\frac{D_{\text {env }}\left(f_{n}\right)}{D_{\text {num }}\left(f_{n}\right)}\right)^{2 / b},
$$

where $G_{m}\left(f_{n}\right)$ is the damage equivalent PSD after $m$ iterations

(7) Calculate the ERS in frequency domain using the updated PSD and Equation (12) and compare with the ERS envelope calculated in time domain

(8) Update the predetermined test time and PSD level to match both the FDS and ERS envelope of the field data 


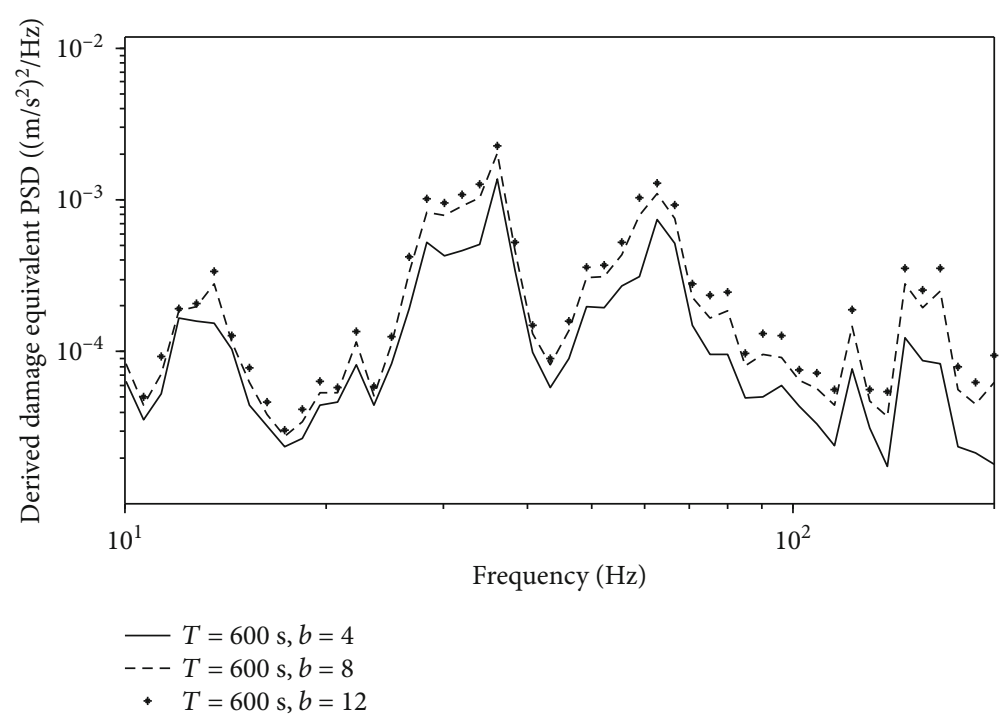

(a)

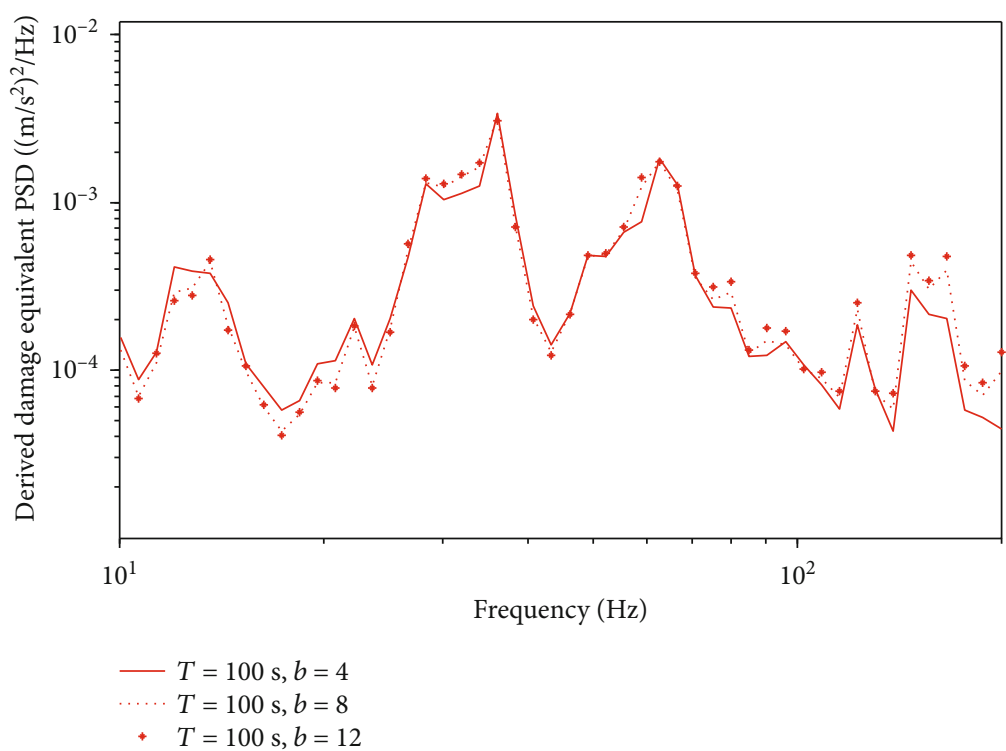

(b)

Figure 8: Continued. 


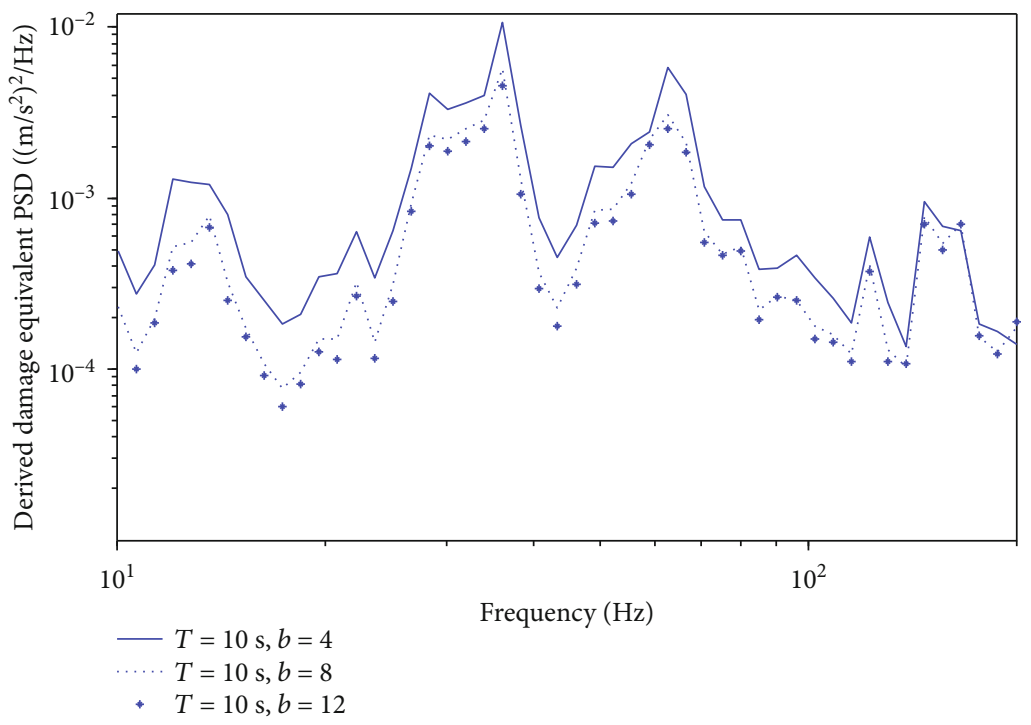

(c)

Figure 8: Equivalent PSD under different test times with different values of $b$ : (a) test time $600 \mathrm{~s}$; (b) test time $100 \mathrm{~s}$; (c) test time 10s.

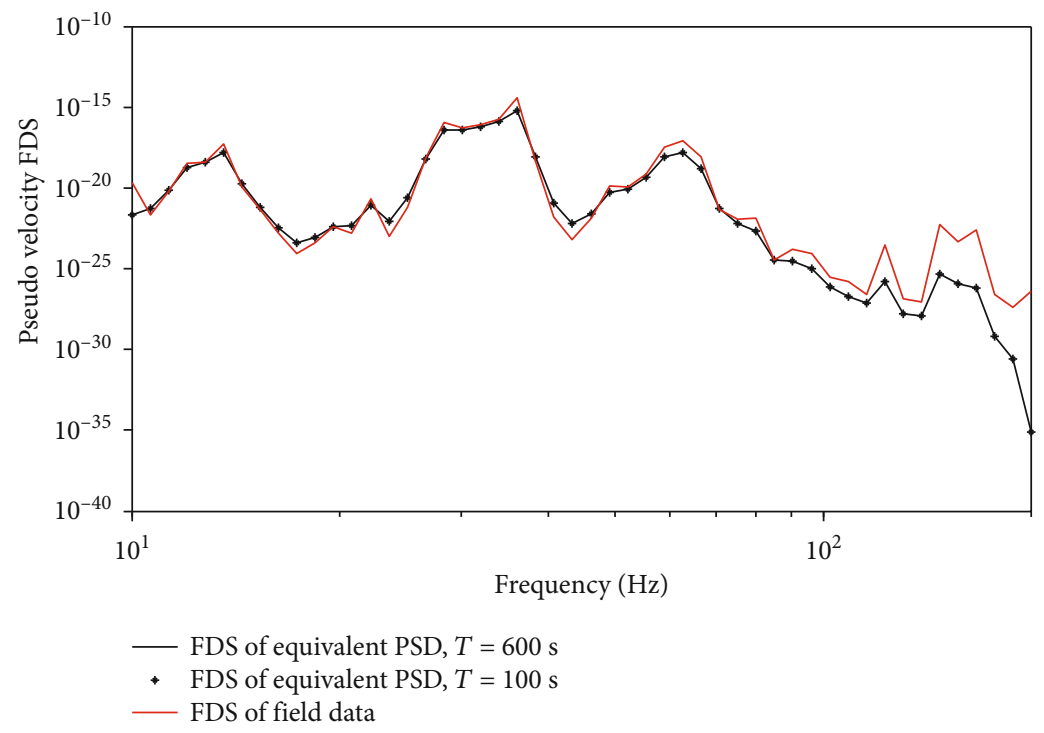

FIgURE 9: Comparison of FDS between field data and equivalent PSD before correction.

\section{Results and Discussion}

3.1. Case Study. Field measured non-Gaussian data are used to demonstrate the procedure in Section 4. The field data is from an Ericsson Mast Project. The random acceleration signal induced by wind is taken from a test item installed on a mast, as shown in Figure 1. The test item is a standard Ericsson Micro Radio Base equipment, manufactured in cast lightweight alloy with integrated heat sinks, equipped with B\&K WB0179 triaxial accelerometer sets and a wind speed measuring device. The dimensions are $530 * 400 * 185 \mathrm{~mm}$, and the weight is about $21 \mathrm{~kg}$. The back side of the box was mounted to the mast at a height of 50 meters.
Acceleration signals were collected using 8 channels, as shown in Figure 2. From Figure 2, we can see that the kurtosis of each signal is greater than 3, indicating the nonGaussianity. To show the error introduced by direct calculation of PSD from the non-Gaussian signal, the data collected from channel 5 is used here as an example. The PSD is calculated, and the Gaussian signal is synthesized, as shown in Figure 3. The PSD and the PDF are compared between the field data and the synthesized Gaussian signal, as shown in Figures 4 and 5, respectively. From Figures 4 and 5, we can see that the PDF of the field data is clearly different from that of the synthesized Gaussian signal, although the PSD are basically the same. The pseudovelocity FDS of the measured 


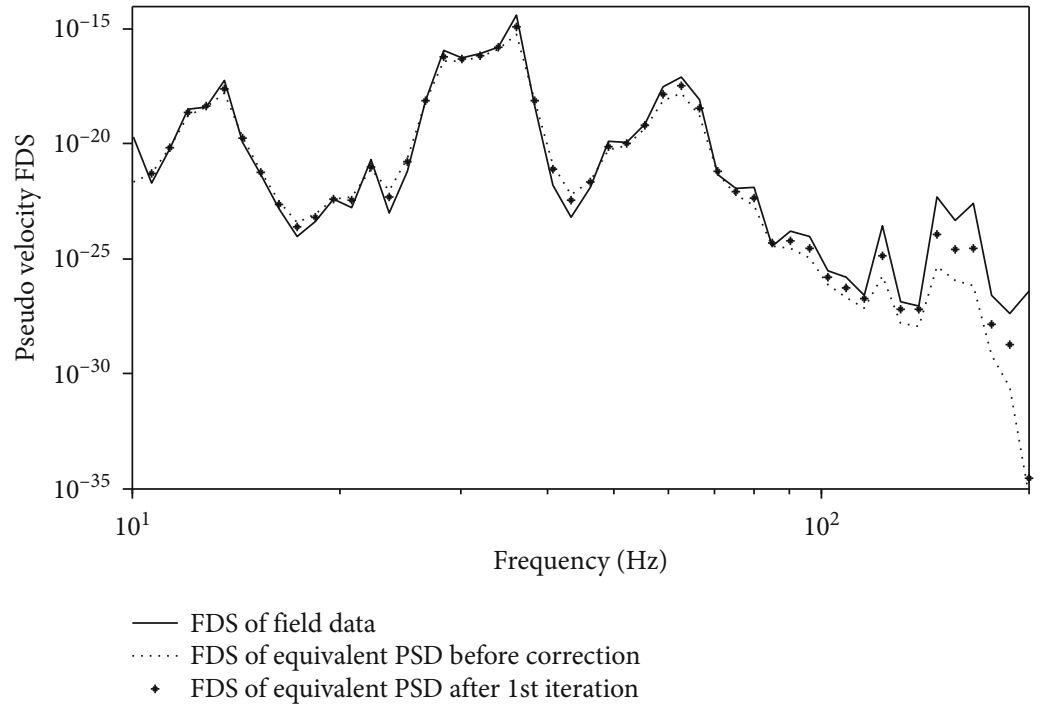

FIgURE 10: Comparison of FDS between field data and equivalent PSD before and after the first correction.

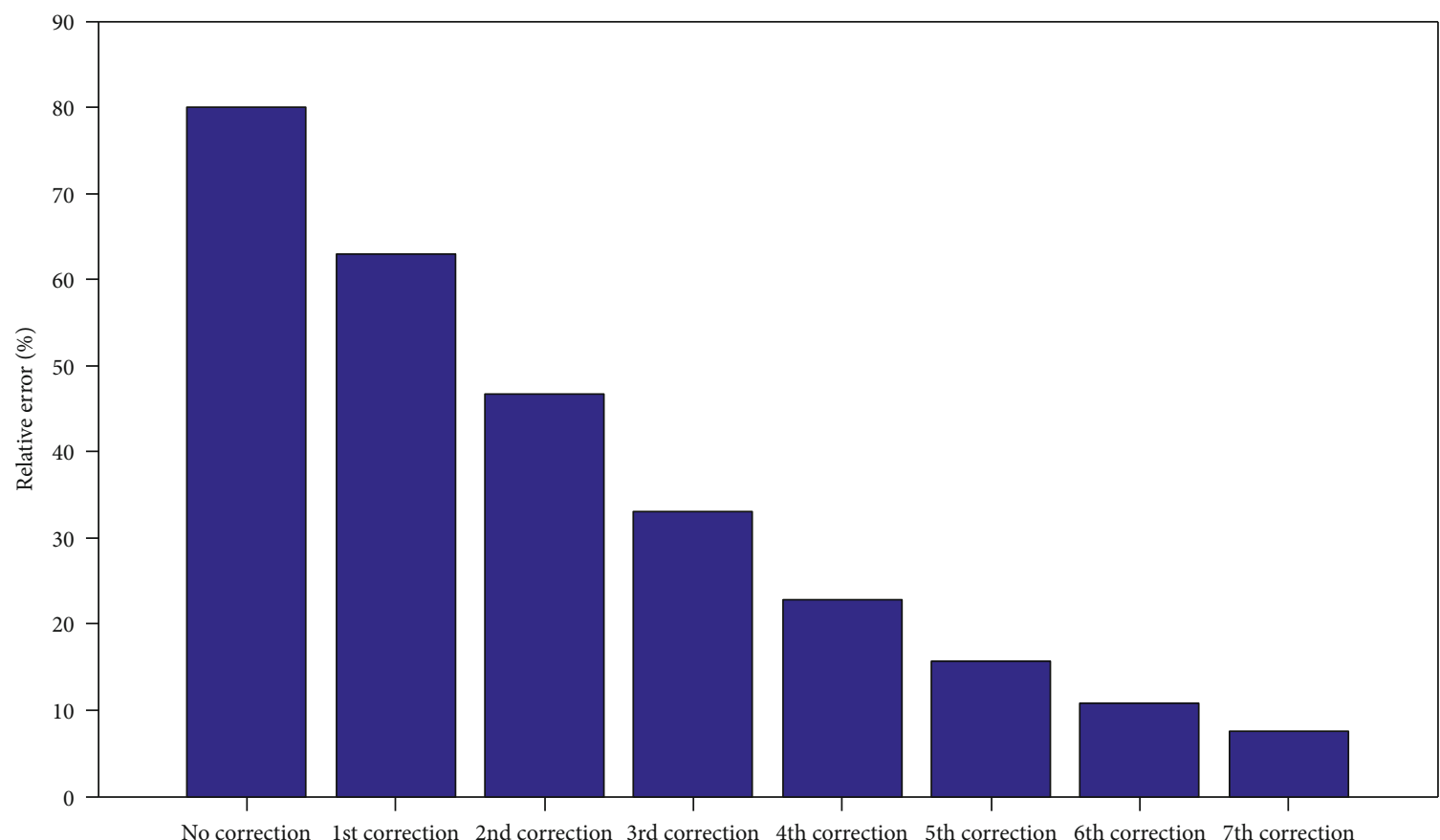

FIGURE 11: FDS error analysis before and after correction.

non-Gaussian signal is significantly higher than that of the synthesized Gaussian load, as shown in Figure 6. From Figures 3-6, we can see that the direct calculation of PSD from a non-Gaussian signal will lead to an obvious undertest.

To determine the effects of damping ratio and fatigue exponent on the calculation of response spectra and PSD synthesis, the pseudovelocity FDS of field data were calculated with different values of $Q(10,25,50)$ and $b(4,8$, 12), as shown in Figure 7. From Figure 7, we can see that the FDS decreases and the dispersion increases, as the value of $b$ increases. The value of $Q$ has little effects on FDS (compared with the effect of $b$ ).
Set the value of $Q$ to 50 , calculate FDS with different values of $b$, and take the envelope. The equivalent PSD under different test times $(T=600 \mathrm{~s}, 100 \mathrm{~s}, 10 \mathrm{~s})$ are derived using the FDS envelope and Equation (10), as shown in Figure 8. From Figure 8, we can see that the equivalent PSD level increases as the test time decreases. The effects of the value of $b$ on the equivalent PSD depend on the predetermined accelerated test time. With $T$ equals to $600 \mathrm{~s}$ (not accelerated), the equivalent PSD increases as the value of $b$ increases. With $T$ equals to $100 \mathrm{~s}$, the value of $b$ has little effect on the equivalent PSD. With $T$ equals to $10 \mathrm{~s}$, however, the equivalent PSD decreases as the value of $b$ increases. 


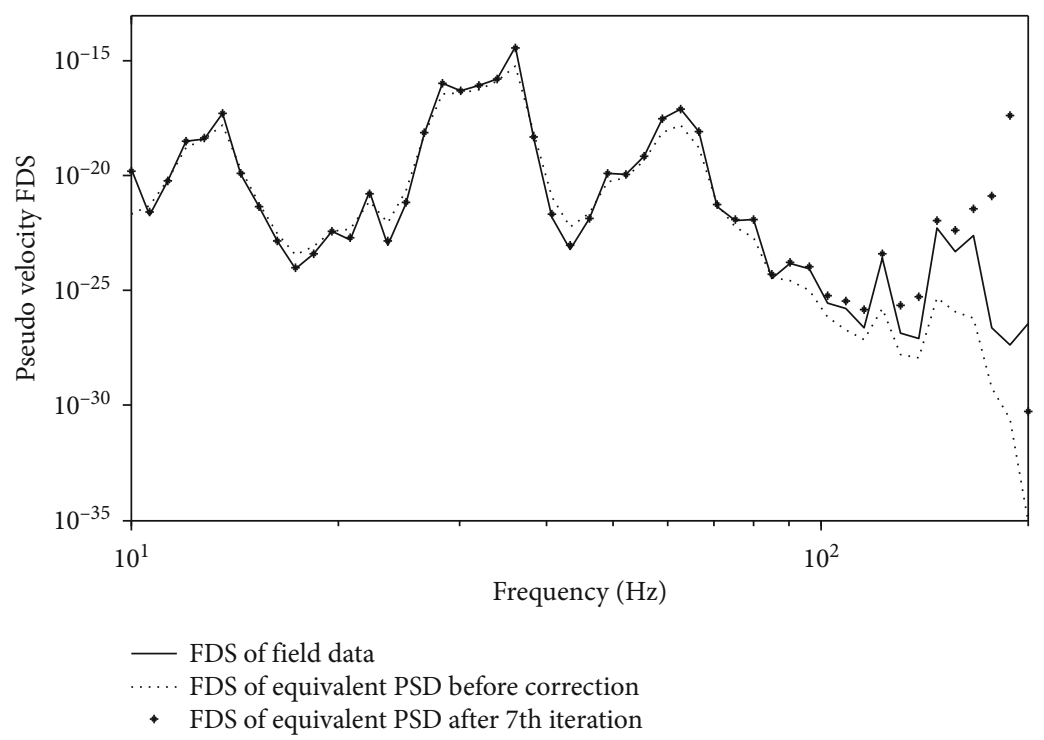

FIGURE 12: Comparison of FDS between field data and equivalent PSD before and after the 7th correction.

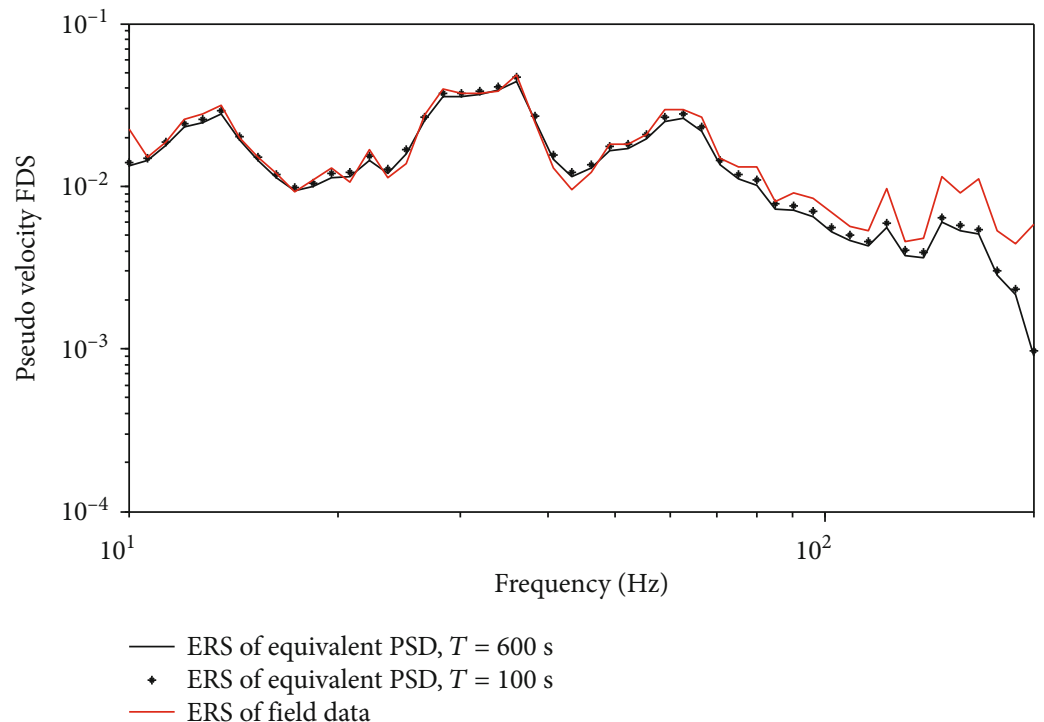

FIgURE 13: Comparison of ERS between equivalent PSD and field data.

The FDS of equivalent PSD for different test times remain the same, as shown in Figure 9. A comparison is made between the FDS of equivalent PSD and nonGaussian field data. Due to the nonsmooth shape of the equivalent PSD, the mismatch is clearly seen, especially within the peak and valley frequency band. To account for the nonsmooth shape, the PSD is updated using Equation (14). A better match between the FDS of the field data and the equivalent PSD is reached after the first iteration, as shown in Figure 10.

The weighted sum of FDS error (see Equation (13)) calculated from the field data and equivalent PSD is reduced from $80.1 \%$ to $7.5 \%$ after 7 iterations. Particularly, the error is close to 0 within the peak and valley frequency band, as shown in Figures 11 and 12. The deviation of PSD between $150 \mathrm{~Hz}$ and $200 \mathrm{~Hz}$ is trivial due to the low level.

A good match between the ERS calculated from the equivalent PSD and that calculated from the field data is shown in Figure 13. Note that although the PSD level can be further increased to reduce the test time, the test item may fail due to the peak response which will not be experienced in the field.

\section{Conclusions}

Considering the effects of dispersion of field data, parameter uncertainties, and the nonsmooth shape of the PSD, on the accuracy of the derived damage equivalent accelerated test 
spectrum, an optimized procedure for PSD synthesis using non-Gaussian data is presented in this paper. Conclusions are as follows:

(1) Direct calculation of PSD from non-Gaussian field data leads to undertest. The envelope of FDS and ERS can be used to derive the damage equivalent PSD, considering the dispersion of multiple nonGaussian field data

(2) Model parameters must be selected dynamically if a priori information is unknown, i.e., the selection of model parameters depends on the predetermined accelerated test time, so as to avoid undertest

(3) The damage equivalent PSD must be updated to account for its nonsmooth shape. The weighted sum of FDS error between field data and derived PSD can be reduced dramatically after only a few iterations. Particularly, the error can be reduced close to 0 within the peak and valley frequency band

\section{Data Availability}

The (MATLAB data file "field data0707.mat") data used to support the findings of this study are available from the corresponding author upon request.

\section{Conflicts of Interest}

The authors declare that there is no conflict of interest regarding the publication of this paper.

\section{Acknowledgments}

This research activity was partially supported by the National Natural Science Foundation of China (Grant No. 52102443) and a project (No. xjr2019036) entitled funding for school-level research projects of Yancheng Institute of Technology.

\section{References}

[1] N. Li, J. Sun, J. Jiao, B. Wu, and C. He, "Quantitative evaluation of micro-cracks using nonlinear ultrasonic modulation method," Ndt \& E International, vol. 79, pp. 63-72, 2016.

[2] Y. Hui, B. Li, H. Kawai, and Q. Yang, "Non-stationary and non-Gaussian characteristics of wind speeds," Wind and Structures, vol. 24, no. 1, pp. 59-78, 2017.

[3] A. Steinwolf, "Vibration testing of vehicle components by random excitations with increased kurtosis," International Journal of Vehicle Noise and Vibration, vol. 11, no. 1, pp. 39-66, 2015.

[4] M. Palmieri, M. Česnik, J. Slavič, F. Cianetti, and M. Boltežar, "Non-Gaussianity and non-stationarity in vibration fatigue," International Journal of Fatigue, vol. 97, pp. 9-19, 2017.

[5] A. Niesłony, M. Bohm, T. Łagoda, and F. Cianetti, "The use of spectral method for fatigue life assessment for non-Gaussian random loads," acta mechanica et automatica, vol. 10, no. 2, pp. 100-103, 2016.
[6] A. Steinwolf, "Random vibration testing with kurtosis control by IFFT phase manipulation," Mechanical Systems and Signal Processing, vol. 28, pp. 561-573, 2012.

[7] J. Zhang, B. Cornelis, B. Peeters, K. Janssens, and P. Guillaume, "A new practical and intuitive method for kurtosis control in random vibration testing," in Proceedings of ISMA 2016 - International Conference on Noise and Vibration Engineering and USD 2016- International Conference on Uncertainty in Structural Dynamics, Leuven, Belgium, 2016.

[8] S. Cui, E. Zheng, and M. Kang, "A time-domain procedure for non-Gaussian stationary environmental testing using zeromemory nonlinear transformation," Journal of Vibration and Control, vol. 26, no. 15-16, pp. 1197-1213, 2020.

[9] X. Fei, L. Chuanri, J. Tongmin, and K. Ahlin, "Synthesis of running RMS-induced non-Gaussian random vibration based on Weibull distribution," Journal of Vibroengineering, vol. 17, no. 7, pp. 3662-3674, 2015.

[10] R. Zheng, H. Chen, D. Vandepitte, and Z. Luo, "Multi-exciter stationary non-Gaussian random vibration test with time domain randomization," Mechanical systems and signal processing, vol. 122, pp. 103-116, 2019.

[11] A. Angeli, B. Cornelis, and M. Troncossi, "Synthesis of Sineon-Random vibration profiles for accelerated life tests based on fatigue damage spectrum equivalence," Mechanical systems and signal processing, vol. 103, pp. 340-351, 2018.

[12] F. Cianetti, A. Alvino, A. Bolognini, M. Palmieri, and C. Braccesi, "On field durability tests of mechanical systems. The use of the Fatigue Damage Spectrum," Procedia Structural Integrity, vol. 3, pp. 176-190, 2017.

[13] M. Bonato and D. Delaux, "Synthesis and validation of accelerated vibration durability tests for engine mounted components," Applied Reliability Durability Conference, Europe, 2018.

[14] C. Lalanne, Mechanical Vibration and Shock Analysis-Volume 5: Specification Development, John Wiley \& Sons, Inc-ISTE, London, 3rd ed. edition, 2014.

[15] J. Wijker, M. Ellenbroek, and A. D. Boer, "Characterization and synthesis of random acceleration vibration specifications," in 4th International Conference on Computational Methods in Structural Dynamics \& Earthquake Engineering, pp. 101-130, Kos Island, Greece, 2013.

[16] M. Decker, S. Kinscherf, N. Bauer, P. David, and M. Serifsoy, "Deriving fatigue equivalent power spectral density spectra for the vibration testing of engine components," Material Science and Engineering Technology, vol. 49, no. 3, pp. 392-405, 2018.

[17] A. Steinwolf and P. Wolfsteiner, "Vibration testing of vehicle components by fatigue damage spectrum control," International Journal of Vehicle Noise and Vibration, vol. 15, no. 2/3, pp. 133-153, 2019.

[18] F. Xu, C. Li, and T. Jiang, "On the shaker simulation of windinduced non-Gaussian random vibration," Shock and Vibration, vol. 2016, Article ID 5450865, 10 pages, 2016.

[19] K. Ahlin, "Comparison of test specifications and measured field data," Sound and Vibration, vol. 40, no. 9, pp. 22-25, 2006.

[20] P. Wolfsteiner, "Fatigue assessment of non-stationary random vibrations by using decomposition in Gaussian portions," International Journal of Mechanical Sciences, vol. 127, pp. 10-22, 2017. 
[21] F. Cianetti, A. Alvino, A. Bolognini, M. Palmieri, and C. Braccesi, "The design of durability tests by fatigue damage spectrum approach," The design of durability tests by fatigue damage spectrum approach. Fatigue and Fracture of Engineering Materials and Structures, vol. 41, no. 4, pp. 787-796, 2018.

[22] P. V. Baren, "The missing knob on your random vibration controller," Sound \& vibration, vol. 39, no. 10, pp. 10-16, 2005.

[23] H. A. Gaberson, "Shock severity estimation," Sound and Vibration, vol. 46, no. 1, pp. 12-20, 2012.

[24] G. R. Henderson and A. G. Piersol, "Fatigue damage related descriptor for random vibration test environments," Sound and Vibration, vol. 29, no. 10, pp. 20-24, 1995. 